\title{
ACTIVATION OF EOSINOPHILS VIA TOLL-LIKE RECEPTOR (TLR)3, TLR7 AND TLR9: LINK BETWEEN VIRAL INFECTION AND ASTHMA?
}

\section{Anne Månsson and Lars Olaf Cardell}

ERS Annual Inflammatory Airway Diseases and Clinical Allergy Award, sponsored by GlaxoSmithKline

Laboratory of Clinical and Experimental Allergy Research, Dept of Otorhinolaryngology, Malmö University Hospital, Lund University, Malmö, Sweden

WINNING ABSTRACT: Asthma is disease characterized by a massive accumulation of eosinophils that release an array of tissue-damaging mediators. Respiratory viral infections are thought to be a leading cause of exacerbations of asthma. One possible explanation might be a direct activation of viral components through Toll-like receptors (TLRs), a receptor family comprising 10 different pathogen-recognizing members (TLR1-TLR10). The virus-recognizing TLRs are TLR3, TLR7/8 and TLR9, which respond to viral dsRNA, ssRNA and CpG-DNA. The present study aimed to investigate the expression of these TLRs and their functions in human eosinophils.

Eosinophils were isolated from peripheral blood using magnetic beads (purity $>\mathbf{9 7 \%}$ ). Cells were incubated with or without poly(I:C), R-837 or CpG alone, the synthetic ligands of TLR3, TLR7 and TLR9, respectively, or combined with IL-4 or histamine. Flow cytometry, and ELISA were used to analyze expression of TLRs and various surface markers, viability and secretion of inflammatory mediators.

Eosinophils expressed proteins for TLR3, TLR7 and TLR9. Poly(I:C), R-837 and CpG prolonged survival, up-regulated expression of the adhesion molecule CD11b and increased secretion of IL-8 compared to unstimulated controls. These effects were affected by the presence of IL-4 and histamine.

This study shows that several viral products directly activate eosinophils through their TLRs. Since eosinophils are central in asthma, the TLR system may be an important mechanism of eosinophil activation linking viral infections with exacerbations. Consequently, this system represents a future clinical target for the resolution of asthmatic disease.

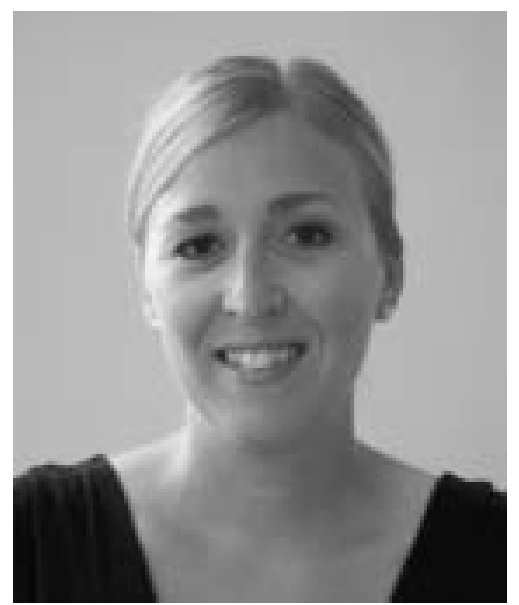

Anne Månsson

Laboratory of Clinical and Experimental Allergy Research, Department of Otorhinolaryngology, Malmö University Hospital UMAS, Lund University, Malmö, Sweden

\section{MY JOB AND THE ROLE OF THE UNIT IN WHICH I WORK}

I work at the Laboratory of Clinical and Experimental Allergy Research at the Dept of Otorhinolaryngology, Malmö University Hospital, Lund University in Malmö, Sweden.

STATEMENT OF INTEREST: None declared.
Our research unit has a long-standing interest in allergy and airway inflammation, with more recent focus on the interaction between the innate and adaptive immunity mediated by the Toll-like receptors (TLRs). The work presented in my abstract constitutes part of my ongoing PhD thesis, entitled "Cellular expression and function of TLRs in upper airway inflammation", which will be completed at the beginning of 2009. As our research unit is situated at the hospital, we are completely integrated with the clinic, which allows us to obtain thoroughly characterised patient material, including blood and bone marrow samples, biopsies, nasal lavage fluids, tonsils and other tissues. This enables our basic scientific findings to be connected into practical and clinical applications, so called "bench-to-bedside". To this end, we are using an array of research techniques, including cell culture, real-time RT-PCR, ELISA, flow cytometry, Western blot, immunohistochemistry, single nucleotide polymorphism genotyping and DNA microarray analysis. Our laboratory believes in the concept of "united airway disease", where the respiratory tract is regarded as a whole and the nose can be used as a model to measure effects on the lungs in patients suffering from allergic rhinitis, asthma or both. For this purpose, we are using techniques, such as rhinomanometry, spirometry and measurements of nitric oxide levels in both the nose and lung.

To ensure translational research, clinicians work together with full-time post docs and PhD students with the help of two technicians, all under the supervision of Professor Lars Olaf Cardell. Among several ongoing projects, at present there are five associated with TLRs, as follows: 1) focuses on finding 
genetic variations in genes involved in allergic rhinitis and asthma, e.g. TLRs and S100A7/psoriasin; 2) centred on expression and in vitro function of TLRs on isolated cells (e.g. neutrophils, eosinophils and different subsets of lymphocytes) from peripheral blood and tonsils; 3 ) working with the effects of inflammatory mediators on airway smooth muscle, using an in vitro model of cultured airways from mouse and guinea pig; 4) uses the nose as a model to investigate how different combinations of anti-inflammatory mediators (e.g. steroids), TLR agonists and allergens affect the lung; and 5) examining how chronic inflammation and TLR stimulation affects normal and malignantly transformed airway epithelial cell lines (A549, Detroit-562, Hep2, Chang and NL-20). My contribution to our research team is my thesis work, in which I investigate how TLRs are expressed and function in different types of leukocytes, and explore their role in upper airway inflammation. I am also involved in supervising undergraduate students.

\section{MY WINNING ABSTRACT AS PART OF MY RESEARCH}

The immune system is traditionally divided into two closely linked parts; the immediate innate immunity and the slower adaptive immunity. The interaction between these two parts is, to a large extent, mediated by TLRs. The overall goal of our research is to enhance the understanding of this interaction and to delineate the role of TLRs and their implication in allergic disease. TLRs are so-called pathogen-recognition receptors, comprising 10 different members (TLR1-TLR10). Each TLR recognises a specific pathogen-associated molecular pattern, including lipopolysaccharide (LPS), bacterial lipoproteins, peptidoglycan, flagellin and different forms of nucleic acids, thereby initiating a host-defence response. Although the TLRs have a protective role to the host, TLR signalling abnormalities and polymorphisms in the TLR genes are increasingly implicated in the pathogenesis of diseases where inflammation is a central feature, such as cancer, autoimmune disorders and allergic diseases [1].

Several studies suggest that respiratory viral infections contribute to allergic sensitisation and development of asthma, and in subjects with already established asthma, viral infections are a common cause of disease exacerbation [2-4]. The exact mechanism behind this is not known, but one possible explanation is virus-induced activation of eosinophils, one of the principal cell types involved in allergy-associated inflammation, through their TLRs. A hallmark of the virus-recognising TLRs is that they are located intracellularly in endosomes and that they respond to nucleic acids from viruses, such as double-stranded RNA (TLR3), single-stranded RNA (TLR7/8) and unmethylated CpG DNA (TLR9). In the context of the respiratory tract, rhinovirus, influenza virus, coronavirus and respiratory syncytial virus are common causes of infection [5]. The work presented in the award-winning abstract is from my latest study, in which this question was addressed. We investigated expression and function of virus-recognising TLRs in eosinophils, and assessed their role in allergic airway inflammation. By analysing magnetic bead-sorted eosinophils (>97\% purity), we found expression of TLR3, TLR7 and TLR9 proteins. All TLRs responded to stimulation by the cognate ligands poly(I:C), R-837 (Imiquimod) and CpG, respectively, to induce upregulated expression of the adhesion molecule CD11b, interleukin (IL)-8 secretion and enhanced cell survival by eosinophils. Furthermore, to investigate if the virus-induced TLR activation is augmented during an allergic reaction, cells were concomitantly stimulated with either histamine or IL-4, two key mediators of allergic inflammation, in order to mimic the allergic inflammation. As expected, an enhanced activation of TLR7 and TLR9 was seen. Also, we wanted to study whether there are differences in responsiveness to TLR stimulation between healthy and allergic individuals. By comparing eosinophils obtained from healthy controls and atopic patients with allergic rhinitis, we found that the allergic individuals responded more robustly to R-837 and CpG than the healthy controls. Taken together, this study shows that several viral products can cause a direct activation of eosinophils via the TLR system and that atopic individuals appear to be more responsive. Therefore, it is tempting to speculate on a role for TLRs on eosinophils in airway infectioninduced asthma exacerbations.

\section{MY RESEARCH AS PART OF MY WORKING GROUP/ RESEARCH TEAM}

Allergic rhinitis and asthma are common diseases in the Western world, and the changes in lifestyle that have occurred during recent decades are likely to contribute to their increased prevalence in developed countries. The aetiology behind these diseases is complex, but the principal mechanisms appear to be the same. Respiratory tract infections, by both bacteria and/or viruses, often precede asthma exacerbations [6]. This phenomenon may comprise several different steps and mechanisms, but an activation of TLRs is clearly one such possible explanation. The relationship between infection and exacerbation of allergic disease has been extensively investigated at our research unit. The results presented in my abstract fit well with a recent study performed at our laboratory by BACHAR et al. [7], where patients with allergic rhinitis (to birch and/or timothy grass pollen) and healthy controls were challenged with LPS, allergen or allergen followed by LPS. The study shows that challenge with LPS, after an allergen-induced allergic inflammation, gives rise to increased symptom scores, leukocyte numbers and enhanced pulmonary nitric oxide levels. This demonstrates how an upper airway infection, represented by nasal LPS (via TLR4), can also affect the lower airways of patients with symptomatic allergic rhinitis. Thus, it might explain how an upper respiratory infection, via interactions with TLRs, can affect initiation, maintenance and progression of asthma [7].

\section{THE IMPACT OF MY WORK ON CLINICAL OR RESEARCH PRACTICE}

The role of the eosinophil in atopic diseases, such as allergic rhinitis and asthma, has been widely disputed during recent decades. From being considered the principal cause of asthma, primarily by the initiation of airway hyperreactivity, the eosinophil was almost forgotten in the allergic arena. During the last couple of years, however, the eosinophil has been resurrected as an important player in allergic diseases by the possible involvement in tissue remodelling and airway inflammation. The present data suggest that eosinophils also play a role in the acute phase of the inflammatory process. In view of this, we strongly believe the eosinophil to be an important therapeutic target for treatment of infection-induced asthma exacerbations. 


\section{REFERENCES}

1 Pandey S, Agrawal DK. Immunobiology of Toll-like receptors: emerging trends. Immunol Cell Biology 2006; 84: 333-341.

2 van Rijt LS, van Kessel $\mathrm{CH}$, Boogaard I, Lambrecht BN. Respiratory viral infections and asthma pathogenesis: a critical role for dendritic cells? J Clin Virol 2005; 34: 161-169.

3 Contoli M, Caramori G, Mallia P, Johnston S, Papi A. Mechanisms of respiratory virus-induced asthma exacerbations. Clin Exp Allergy 2005; 35: 137-145.

4 Micillo E, Bianco A, D'Auria D, Mazzarella G, Abbate GF. Respiratory infections and asthma. Allergy 2000; 55: Suppl. 61, 42.
5 Papadopoulos NG, Xepapadaki P, Mallia P, et al. Mechanisms of virus-induced asthma exacerbations: stateof-the-art. A GA2LEN and InterAirways document. Allergy 2007; 62: 457-470.

6 Nagase H, Okugawa S, Ota Y, et al. Expression and function of Toll-like receptors in eosinophils: activation by Toll-like receptor 7 ligand. J Immunol 2003; 171: 3977-3982.

7 Bachar O, Gustavsson J, Jansson L, Adner M, Cardell L-O. LPS administration to the allergic nose contributes to lower airway inflammation. Clin Exp Allergy 2007; 37: 1773-1780. 